\title{
STUDY OF IMPULSIVITY AND SUICIDE INTENT IN ATTEMPTED SUICIDE PATIENTS
}

\author{
A. NiranjanaDevi1, R. Muralitharan²
}

1Professor, Department of Psychiatry, M. G. Memorial Government Hospital (MGMGH) and KAPV Government Medical College, Tiruchirappalli, Tamilnadu.

${ }^{2}$ Associate Professor, Department of Psychiatry, M. G. Memorial Government Hospital (MGMGH) and KAPV Government Medical College, Tiruchirappalli, Tamilnadu. \footnotetext{
ABSTRACT

BACKGROUND

Suicide is one of the leading cause of death, both in developing and developed countries. Nowadays suicidal behaviour has been recognised as an increasing public health problem.

The aim of this study is to study the impulsivity and suicidal intent in suicide attempters.

The objectives of this study are-

1. To assess the impulsivity in suicide attempters.

2. To evaluate the suicide intent among the attempters.

3. To identify the relationship between impulsivity and intent among suicide attempters.

Setting- Study was done in Department of Psychiatry, Mahatma Gandhi Memorial Hospital attached to KAPV Government Medical College, Trichy.

Design- This is a cross-sectional observational study.
}

\section{MATERIALS AND METHODS}

One hundred suicide attempters above the age of 18, attending Department of Psychiatry were included in the study. Kuppuswamy's revised socioeconomic status scale, Barratt Impulsiveness Scale (BIS-II) and Beck's suicide intent scale were administered for study population.

Statistical Analysis- Data were analysed using appropriate statistical methods such as ANOVA, Chi-Square test and Pearson correlation coefficient.

\section{RESULTS}

Among one hundred study population, almost equal percentage of male and female were present and majority were less than age of 45 years. In this study, statistically significant association between persons with family history of mental illness and suicidal intent and impulsivity were made out. Family history of suicide attempt did not show significant association neither to suicidal intent nor to impulsivity. Religious status had significant association with suicidal impulsivity.

\section{CONCLUSION}

Even though there is a difference among suicide attempters, there is significant difference among various impulsivity factors between gender. Socio-demographic factors except religion revealed no correlation for impulsivity and suicidal intent.

\section{KEYWORDS}

Impulsivity, Intent, Suicide, Mental Illness.

HOW TO CITE THIS ARTICLE: NiranjanaDevi A, Muralitharan R. Study of impulsivity and suicide intent in attempted suicide patients. J. Evolution Med. Dent. Sci. 2017;6(90):6333-6339, DOI: 10.14260/jemds/2017/1378

\section{BACKGROUND}

Suicide is to the psychiatrist as cancer is to the internist. Suicidal behaviour is a major public health problem nowadays. ${ }^{2}$ Suicide is the leading cause of premature death, especially among young adults. In many westernised countries, suicide is the frequent cause of death than traffic accidents. Globally, one person commits suicide every minute. ${ }^{3}$ A process which leads to suicidal ideation, suicidal communication and self-destructive behaviour in some cases

'Financial or Other Competing Interest': None.

Submission 06-10-2017, Peer Review 02-11-2017,

Acceptance 09-11-2017, Published 20-11-2017.

Corresponding Author:

Dr. R. Muralitharan,

Associate Professor

Department of Psychiatry,

M. G. Memorial Government Hospital (MGMGH) and

KAPV Government Medical College,

Tiruchirappalli-600017, Tamilnadu.

E-mail: drmuramind@gmail.com

DOI: $10.14260 /$ jemds $/ 2017 / 1378$ even to the suicide and its consequences to the survivors is referred to as suicidal process. ${ }^{3}$ Suicide is considered as fatal act of self-injury undertaken with more or minimal intent, however vague or ambiguous. ${ }^{4}$

Suicide is mostly preceded by suicidal behaviour or feelings, plans as well as warnings. In about half of suicides, history of previous attempt is found and this is an opportunity for suicide intervention.

From various viewpoints about suicide, culture defines basic attitude towards life and death. We still have stigma against suicide. Religion is still a meaningful and protective factor for many individuals in a crisis of suicide. ${ }^{3}$

Sociology view suicide as a social phenomenon. Suicides are viewed as disturbance of relationship between society and individuals. Psychologically, Freud's theory assumes that self-destructive behaviour in depression represents aggression directed against self. Suicidal behaviour is highly familial. Heritability of suicidal behaviour appears to be $30 \%$ - $50 \%$ and interactions with various environmental factors is significant. ${ }^{3}$ 
Usually, suicide has no single cause. In this, several interacting risk factors can be identified.

Socio-demographic variables such as male gender, elderly age group, low socioeconomic and lower educational status, unmarried or divorced, living alone and unemployment are the risk factors of suicide. Virtually, all mental disorders carry an increased risk of suicide. The most common psychiatric diagnosis in suicide are major depression and alcohol dependence followed by personality disorders and schizophrenia. ${ }^{2}$

The risk of completed suicide is highest among patients with both major depression and substance use disorders. ${ }^{5}$ Individuals with problems in help seeking, social communication, self-disclosure also pose a suicide risk as do personality traits of aggression and impulsivity. ${ }^{2}$ Mechanisms underlying some risk factors (aggression, impulsivity, suicide intent) remain unclear.

Suicide intent is defined as seriousness of the wish of a patient to end his life. ${ }^{6}$ Suicide attempters with high intent scores may carry higher risk of completed suicide, although not all studies support this view. ${ }^{7}$

Suicide intent was measured via hopelessness. Hopelessness is a cognitive trait associated with suicide, leading suicidal patients to believe that suicide is the only feasible strategy for dealing with their seemingly insoluble problems. ${ }^{8}$ Hopelessness is the mediating variable between depression and suicide intent, 9 but all findings are not consistent with this interpretation. ${ }^{10,11}$

Individuals who choose the more immediate reward are often said to be impulsive. Action without planning or thinking about its consequences is central to most definitions of impulsivity. It may also be defined as a tendency to respond quickly without understanding its consequences. ${ }^{12}$ Thus, impulsivity appears to be associated with a failure of behavioural filtering process outside of consciousness ${ }^{13}$ with compromised ability to use knowledge to guide behaviour. ${ }^{14}$ Impulsive individuals have inappropriately short threshold for response, lack of reflection, lack of modulation and lack of potential gain.

Impulsivity is seen as a complex, multidimensional trait that is viewed both as a normal dimensional behaviour and as core pathological features of mental disorders such as Attention Deficit Hyperactivity Disorder, Suicidal and aggressive behaviour, antisocial and borderline personality disorder, alcohol and drug dependence. ${ }^{15}$

Baumeister thought suicide as a means of escape from aversive self-awareness. To alleviate this aversive selfawareness, they achieve a state of "cognitive deconstruction." This is characterised by a constricted, present focused time perspective and cognitive rigidity. This deconstructed state dulls self-awareness and leads to disinhibition. Due to this disinhibition, individuals attempt suicide impulsively. 16

Impulsivity is considered as a state impulsivity (impulsivity experienced at particular point of time), trait impulsivity (variable that influences involvement in risky behaviour). It has been stated that state impulsivity is less important than impulsivity exhibited throughout the life. ${ }^{17}$ In previous studies, it is documented that persons who attempt suicide impulsively are less depressive and have a less strong intent to die than non-impulsive attempters. 4

\section{Aim}

To study the impulsivity and suicidal intent in suicide attempters.

\section{Objectives}

1. To assess the impulsivity in suicide attempters.

2. To evaluate the suicide intent among the attempters.

3. To identify the relationship between impulsivity and intent among suicide attempters.

\section{MATERIALS AND METHODS}

One hundred attempted suicide patients attending Department of Psychiatry, Mahathma Gandhi Memorial Government Hospital (MGMGH) attached to K.A.P.V. Govt. Medical College, Tiruchirapalli, were included in this study.

\section{Ethical Clearance}

Ethical clearance for this study was obtained from college ethical committee. This study involved cross-sectional observational and purposive sampling procedure. Every patient was assessed once for the study.

\section{Materials}

Suicide attempters above the age of 18 years, who were referred from medical wards after the improvement of their general condition. Patients with cognitive impairment, poor physical status were excluded from the study. Informed consent was obtained from them.

\section{Methods}

Individual's details were collected using socio-demographic data sheet and Kuppuswamy's socioeconomic status scale was applied to all subjects.

Barratt Impulsiveness scale18 (BIS-II) is a 30 -item questionnaire, includes three second order factors labelled Attentional (F1), Non-planning (F2), Motor (F3). Factor (F1) combined first order factors attention and cognitive instability impulsiveness, the second factor (F2) non-planning was defined by perseverance and motor, while the third factor (F3) is the combination of first order factors such as selfcontrol and cognitive complexity.

A total score of 72 and above indicates high impulsivity, scores between 52 and 71 is defined as within the normal limits of impulsiveness. Scores below 52 are extremely over controlled.

\section{Beck's Suicide Intent Scale}

This is a 15 -item questionnaire, each item is scored from 0 to 2 giving a possible range of score from 0 to 30. It consists of two parts: part 1 (items 1 to 8 ) covers the objective circumstances of the attempt, part 2 (items 9 to 15) reflects the attempter's self-report. This scale assesses the severity of the subject's psychological intent to die at the time of attempt. 6

Descriptive statistics for socio-demographic profile: Chisquare test was done to analyse the qualitative variables, ANOVA test was applied for comparing various dimensions of suicidal impulsivity score and Pearson correlation test was done to compare the relation between impulsivity and suicide intent. 


\section{RESULTS}

Among study population of 100 , females constitute $51 \%$ $(\mathrm{n}=51)$ and males constitute $49 \%(\mathrm{n}=49)$. Majority $(\mathrm{n}=87)$ were below the age of 45 years and nearly half of the population $(n=53)$ were married. In this study, two-third $(n=70)$ of them were from rural area and joint family setup; $88 \%$ of Hindus and $7 \%$ of Muslims constitute study population (Table 1).

In evaluating suicide intent, $75 \%(n=75)$ expressed medium suicide intent and $19 \%$ had high intent. Among females, $78 \%$ expressed medium suicide intent and 14\% expressed high suicide intent. In males, $71 \%$ had medium intent and $24 \%$ had high intent. High suicide intent was more in males (24\%) than females (14\%). Even though there was a difference in expressing suicide intent between males and females, it was not statistically significant (Table 2).

In comparing the suicide intent among various age groups, persons between 31 - 45 yrs. showed high intent (24\%) followed by 18 - 30 yrs. (17\%). All the people above 45 yrs. had medium-to-high suicide intent. In this study, marital status did not make any significant difference in suicide intent. Educational qualification and socio-economic status had no impact on suicide intent (Table 2).

In our study, $38 \%$ of persons with family history of psychiatric illness expressed high suicide intent and this is statistically significant $(\mathrm{p}=0.015)$. Family history of suicide attempt did not show association with suicide intent (Table 3).

In analysing impulsivity, around three-fourths of the study population scored within normal limits of impulsivity. Around one-fifth of the study population scored high on impulsivity. Among the people with normal limits of impulsiveness, females constitute $56 \%$ and males $44.3 \%$. Within high impulsivity group, males comprised $63 \%$ and females $37 \%$. No significant association was found in analysing impulsivity with living status, education, occupation and socioeconomic status (Table 4).

There is significant association between impulsivity and religious status of study members $(\mathrm{p}=0.007)$.

Persons with family history of psychiatric illness had significant association with impulsivity in comparing persons without family history of psychiatric illness $(p=0.04)$. When analysing persons with and without family history of suicide attempt, no difference was made out regarding impulsivity (Table 5).

There was no statistical significance in assessing impulsivity in individuals with mental illness (depressive disorder, alcohol dependence syndrome, impulse control disorder, adjustment disorder with depressed mood) and individuals without history of mental illness (Table 5).
In evaluating factors of impulsivity among male and female participants, males showed statistical significance in self-control factor $(p=0.055)$, females showed statistical significance in $1^{\text {st }}$ order factor motor $(p=0.027)$ (Table 6).

\begin{tabular}{|c|c|c|c|}
\hline Sl. No. & & Total & \\
\hline & & $(n=100)$ & $-100 \%$ \\
\hline \multirow[t]{4}{*}{1} & Age & & \\
\hline & $18-30$ yrs. & 66 & $66.0 \%$ \\
\hline & $31-45$ yrs. & 21 & $21.0 \%$ \\
\hline & $46-60$ yrs. & 13 & $13.0 \%$ \\
\hline \multirow[t]{3}{*}{2} & Sex & & \\
\hline & Male & 49 & $49.0 \%$ \\
\hline & Female & 51 & $51.0 \%$ \\
\hline \multirow[t]{5}{*}{3} & Marital Status & & \\
\hline & Never Married & 44 & $44.0 \%$ \\
\hline & Married & 53 & $53.0 \%$ \\
\hline & Divorced/ Separated & 1 & $1.0 \%$ \\
\hline & Widow & 2 & $2.0 \%$ \\
\hline \multirow[t]{3}{*}{4} & Living Status & & \\
\hline & Nuclear Family & 70 & $70.0 \%$ \\
\hline & Joint Family & 30 & $30.0 \%$ \\
\hline \multirow[t]{3}{*}{5} & Area of Living & & \\
\hline & Rural & 70 & $70.0 \%$ \\
\hline & Urban & 30 & $30.0 \%$ \\
\hline \multirow[t]{4}{*}{6} & Religion & & \\
\hline & Hindu & 88 & $88.0 \%$ \\
\hline & Muslim & 7 & $7.0 \%$ \\
\hline & Christian & 5 & $5.0 \%$ \\
\hline \multirow[t]{7}{*}{7} & Education & & \\
\hline & Illiterate & 9 & $9.0 \%$ \\
\hline & Primary & 25 & $25.0 \%$ \\
\hline & Middle & 26 & $26.0 \%$ \\
\hline & HSC & 24 & $24.0 \%$ \\
\hline & Diploma & 7 & $7.0 \%$ \\
\hline & $\begin{array}{l}\text { Graduate and } \\
\text { Postgraduate }\end{array}$ & 9 & $9.0 \%$ \\
\hline \multirow[t]{7}{*}{8} & Occupation & & \\
\hline & Unemployed & 3 & $3.0 \%$ \\
\hline & Unskilled & 8 & $8.0 \%$ \\
\hline & Semiskilled & 49 & $49.0 \%$ \\
\hline & Skilled & 37 & $37.0 \%$ \\
\hline & $\begin{array}{c}\text { Clerical and Shop } \\
\text { Owner }\end{array}$ & 1 & $1.0 \%$ \\
\hline & Semi Professional & 2 & $2.0 \%$ \\
\hline \multirow[t]{5}{*}{9} & Socioeconomic Status & & \\
\hline & Upper Middle & 3 & $3.0 \%$ \\
\hline & Middle & 34 & $34.0 \%$ \\
\hline & Lower Upper & 57 & $57.0 \%$ \\
\hline & Lower & 6 & $6.0 \%$ \\
\hline
\end{tabular}

\begin{tabular}{|c|c|c|c|c|c|c|c|c|c|}
\hline \multirow{2}{*}{$\begin{array}{l}\text { Sl. } \\
\text { No. }\end{array}$} & \multirow{2}{*}{\multicolumn{2}{|c|}{ Demographic Factors }} & \multicolumn{3}{|c|}{ Suicide Intent Score } & \multirow{2}{*}{$\begin{array}{c}\text { Total } \\
(\mathrm{N}=100)\end{array}$} & \multicolumn{3}{|c|}{ Chi-Square Test } \\
\hline & & & $\begin{array}{c}\text { Low } \\
(n=6)\end{array}$ & $\begin{array}{c}\text { Medium } \\
(n=75)\end{array}$ & $\begin{array}{c}\text { High } \\
(n=19)\end{array}$ & & Value & df & Significance \\
\hline \multirow{2}{*}{1.} & \multirow{2}{*}{ Sex } & Male & 2 & 35 & 12 & 49 & \multirow{2}{*}{2.277} & \multirow{2}{*}{2} & \multirow{2}{*}{0.320} \\
\hline & & Female & 4 & 40 & 7 & 51 & & & \\
\hline \multirow{3}{*}{2.} & \multirow{3}{*}{ Age } & 18 - 30 yrs. & 4 & 51 & 11 & 66 & \multirow{3}{*}{2.020} & \multirow{3}{*}{4} & \multirow{3}{*}{0.732} \\
\hline & & $31-45$ yrs. & 2 & 14 & 5 & 21 & & & \\
\hline & & $46-60$ yrs. & 0 & 10 & 3 & 13 & & & \\
\hline 3. & Marital Status & Unmarried & 3 & 33 & 8 & 44 & 5.007 & 6 & 0.543 \\
\hline
\end{tabular}




\begin{tabular}{|c|c|c|c|c|c|c|c|c|c|}
\hline & & Married & 3 & 40 & 10 & 53 & & & \\
\hline & & Divorced/ Separated & 0 & 0 & 1 & 1 & & & \\
\hline & & Widowhood & 0 & 2 & 0 & 2 & & & \\
\hline \multirow{2}{*}{4.} & \multirow{2}{*}{ Living Status } & Nuclear & 3 & 53 & 14 & 70 & \multirow{2}{*}{1.282} & \multirow{2}{*}{2} & \multirow{2}{*}{0.527} \\
\hline & & Joint & 3 & 22 & 5 & 30 & & & \\
\hline \multirow{2}{*}{5.} & \multirow{2}{*}{ Area of Living } & Rural & 5 & 52 & 13 & 70 & \multirow{2}{*}{0.546} & \multirow{2}{*}{2} & \multirow{2}{*}{0.761} \\
\hline & & Urban & 1 & 23 & 6 & 30 & & & \\
\hline \multirow{3}{*}{6.} & \multirow{3}{*}{ Religion } & Hindu & 5 & 67 & 16 & 88 & \multirow{3}{*}{2.566} & \multirow{3}{*}{4} & \multirow{3}{*}{0.633} \\
\hline & & Muslim & 1 & 5 & 1 & 7 & & & \\
\hline & & Christian & 0 & 3 & 2 & 5 & & & \\
\hline \multirow{4}{*}{7.} & \multirow{4}{*}{$\begin{array}{l}\text { Socio- } \\
\text { Economic } \\
\text { Status }\end{array}$} & Upper Middle & 0 & 2 & 1 & 3 & \multirow{4}{*}{3.661} & \multirow{4}{*}{6} & \multirow{4}{*}{0.722} \\
\hline & & Middle & 2 & 27 & 5 & 34 & & & \\
\hline & & Lower Upper & 4 & 40 & 13 & 57 & & & \\
\hline & & Lower & 0 & 6 & 0 & 6 & & & \\
\hline & & Table 2. Associat & & Dem & c $S t$ & St & ntent & & \\
\hline
\end{tabular}

\begin{tabular}{|c|c|c|c|c|c|c|c|c|c|}
\hline \multirow{2}{*}{$\begin{array}{c}\text { Sl. } \\
\text { No. }\end{array}$} & \multirow{2}{*}{\multicolumn{2}{|c|}{ Mental Illness }} & \multicolumn{3}{|c|}{ Suicide Intent Score } & \multirow{2}{*}{$\begin{array}{c}\text { Total } \\
(\mathrm{N}=100)\end{array}$} & \multicolumn{3}{|c|}{ Chi-Square Test } \\
\hline & & & \multirow{2}{*}{$\begin{array}{c}\begin{array}{c}\text { Low } \\
(\mathrm{n}=6)\end{array} \\
5\end{array}$} & \multirow{2}{*}{$\begin{array}{c}\begin{array}{c}\text { Medium } \\
(\mathrm{n}=75)\end{array} \\
70\end{array}$} & \multirow{2}{*}{$\begin{array}{c}\begin{array}{c}\text { High } \\
(\mathbf{n}=19)\end{array} \\
17\end{array}$} & & \multirow{3}{*}{\begin{tabular}{|l|} 
Value \\
0.958 \\
\end{tabular}} & \multirow{3}{*}{$\frac{\mathbf{d f}}{2}$} & \multirow{3}{*}{$\begin{array}{c}\begin{array}{c}\text { Significanc } \\
\text { e }\end{array} \\
0.619\end{array}$} \\
\hline \multirow{2}{*}{1.} & \multirow{2}{*}{ Depressive episode } & Without illness & & & & 92 & & & \\
\hline & & With illness & 1 & 5 & 2 & 8 & & & \\
\hline \multirow{2}{*}{2.} & \multirow{2}{*}{$\begin{array}{l}\text { Adjustment disorder with } \\
\text { depressed mood }\end{array}$} & Without illness & 6 & 71 & 16 & 93 & \multirow{2}{*}{3.026} & \multirow{2}{*}{2} & \multirow{2}{*}{0.220} \\
\hline & & With illness & 0 & 4 & 3 & 7 & & & \\
\hline \multirow{2}{*}{3.} & \multirow{2}{*}{ Binge drinking } & Without illness & 6 & 72 & 18 & 96 & \multirow{2}{*}{0.329} & \multirow{2}{*}{2} & \multirow{2}{*}{0.848} \\
\hline & & With illness & 0 & 3 & 1 & 4 & & & \\
\hline \multirow{2}{*}{4.} & \multirow{2}{*}{ Impulse control disorder } & Without illness & 6 & 73 & 19 & 98 & \multirow{2}{*}{0.680} & \multirow{2}{*}{2} & \multirow{2}{*}{0.712} \\
\hline & & With illness & 0 & 2 & 0 & 2 & & & \\
\hline \multirow{2}{*}{5.} & \multirow{2}{*}{ Borderline personality traits } & Without illness & 5 & 67 & 17 & 89 & \multirow{2}{*}{0.210} & \multirow{2}{*}{2} & \multirow{2}{*}{0.900} \\
\hline & & With illness & 1 & 8 & 2 & 11 & & & \\
\hline \multirow{2}{*}{6.} & \multirow{2}{*}{$\begin{array}{l}\text { Multiple psychiatric } \\
\text { disorders }\end{array}$} & Without illness & 6 & 72 & 18 & 96 & & & \\
\hline & & With illness & 0 & 3 & 1 & 4 & 0.329 & 2 & 0.848 \\
\hline & Family history of psychiatric & Without family history & 4 & 72 & 16 & 92 & & & 0.015 \\
\hline 7. & illness & With family history & 2 & 3 & 3 & 8 & 8.429 & 2 & (significant) \\
\hline 8 & Family history of suicide & Without family history & 6 & 71 & 18 & 95 & & & \\
\hline 8. & attempt & With family history & 0 & 4 & 1 & 5 & 0.336 & 2 & 0.845 \\
\hline & & 3. Association betwee & Psyc & ic IIIn & dSui & tent & & & \\
\hline
\end{tabular}

\begin{tabular}{|c|c|c|c|c|c|c|c|c|c|}
\hline \multirow{2}{*}{$\begin{array}{l}\text { Sl. } \\
\text { No. }\end{array}$} & \multirow{2}{*}{\multicolumn{2}{|c|}{ Demographic Factors }} & \multicolumn{3}{|c|}{ Barratt Impulsiveness Score } & \multirow{2}{*}{$\begin{array}{c}\text { Total } \\
(\mathrm{N}=100)\end{array}$} & \multicolumn{3}{|c|}{ Chi-Square Test } \\
\hline & & & $\begin{array}{c}\text { Good } \\
(n=11)\end{array}$ & $\begin{array}{c}\text { Normal } \\
(n=70)\end{array}$ & $\begin{array}{c}\text { High } \\
(n=19)\end{array}$ & & Value & df & Significance \\
\hline \multirow{2}{*}{1.} & \multirow[b]{2}{*}{ Sex } & Male & 6 & 31 & 12 & 49 & \multirow{2}{*}{2.282} & \multirow{2}{*}{2} & \multirow{2}{*}{0.320} \\
\hline & & Female & 5 & 39 & 7 & 51 & & & \\
\hline \multirow{4}{*}{2.} & \multirow{4}{*}{ Marital Status } & Unmarried & 8 & 29 & 7 & 44 & \multirow{4}{*}{5.441} & \multirow{4}{*}{6} & \multirow{4}{*}{0.489} \\
\hline & & Married & 3 & 38 & 12 & 53 & & & \\
\hline & & Divorced/ Separated & 0 & 1 & 0 & 1 & & & \\
\hline & & Widowhood & 0 & 2 & 0 & 2 & & & \\
\hline \multirow{2}{*}{3.} & \multirow{2}{*}{ Living Status } & Nuclear & 7 & 49 & 14 & 70 & \multirow{2}{*}{0.335} & \multirow{2}{*}{2} & \multirow{2}{*}{0.846} \\
\hline & & Joint & 4 & 21 & 5 & 30 & & & \\
\hline \multirow{2}{*}{4.} & \multirow{2}{*}{ Area of Living } & Rural & 8 & 50 & 12 & 70 & \multirow{2}{*}{0.531} & \multirow{2}{*}{2} & \multirow{2}{*}{0.767} \\
\hline & & Urban & 3 & 20 & 7 & 30 & & & \\
\hline \multirow{3}{*}{5.} & \multirow{3}{*}{ Religion } & Hindu & 8 & 63 & 17 & 88 & \multirow{3}{*}{14.129} & \multirow{3}{*}{4} & \multirow{3}{*}{$\begin{array}{c}0.007 \\
\text { (Significant) }\end{array}$} \\
\hline & & Muslim & 0 & 6 & 1 & 7 & & & \\
\hline & & Christian & 3 & 1 & 1 & 5 & & & \\
\hline \multirow{4}{*}{6.} & \multirow{4}{*}{$\begin{array}{c}\text { Socio-Economic } \\
\text { Status }\end{array}$} & Upper Middle & 0 & 3 & 0 & 3 & \multirow{4}{*}{10.938} & \multirow{4}{*}{6} & \\
\hline & & Middle & 8 & 18 & 8 & 34 & & & \\
\hline & & Lower Upper & 3 & 44 & 10 & 57 & & & 0.090 \\
\hline & & Lower & 0 & 5 & 1 & 6 & & & \\
\hline & & ble 4. Association b & en Soci & emog & ic Fact & and Im & sivity & & \\
\hline
\end{tabular}




\begin{tabular}{|c|c|c|c|c|c|c|c|c|c|}
\hline \multirow{2}{*}{$\begin{array}{l}\text { Sl. } \\
\text { No. }\end{array}$} & \multirow{2}{*}{\multicolumn{2}{|c|}{ Mental Illness }} & \multicolumn{3}{|c|}{ Barratt Impulsiveness Score } & \multirow{4}{*}{$\begin{array}{c}\begin{array}{c}\text { Total } \\
(\mathbf{N}=\mathbf{1 0 0})\end{array} \\
92 \\
8\end{array}$} & \multicolumn{3}{|c|}{ Chi-Square Test } \\
\hline & & & $\begin{array}{c}\text { Good } \\
(n=11)\end{array}$ & $\begin{array}{c}\text { Normal } \\
(n=70)\end{array}$ & $\begin{array}{c}\text { High } \\
(n=19)\end{array}$ & & Value & df & Significance \\
\hline \multirow{2}{*}{1.} & \multirow{2}{*}{ Depressive episode } & Without illness & 10 & 64 & 68 & & \multirow{2}{*}{0.242} & \multirow{2}{*}{2} & \multirow{2}{*}{0.886} \\
\hline & & With illness & 1 & 6 & 1 & & & & \\
\hline \multirow[b]{2}{*}{2.} & \multirow{2}{*}{$\begin{array}{c}\text { Adjustment disorder } \\
\text { with depressed } \\
\text { mood }\end{array}$} & Without illness & 10 & 64 & 19 & 93 & \multirow[b]{2}{*}{1.770} & \multirow[b]{2}{*}{2} & \multirow[b]{2}{*}{0.413} \\
\hline & & With illness & 1 & 6 & 0 & 7 & & & \\
\hline \multirow[b]{2}{*}{3.} & \multirow{2}{*}{$\begin{array}{c}\text { Alcohol dependence } \\
\text { syndrome }\end{array}$} & Without illness & 10 & 63 & 16 & 89 & \multirow{2}{*}{0.558} & \multirow{2}{*}{2} & \multirow{2}{*}{0.757} \\
\hline & & With illness & 1 & 7 & 3 & 11 & & & \\
\hline \multirow{2}{*}{4.} & \multirow{2}{*}{ Binge drinking } & Without illness & 10 & 69 & 17 & 96 & \multirow{2}{*}{4.055} & \multirow{2}{*}{2} & \multirow{2}{*}{0.132} \\
\hline & & With illness & 1 & 1 & 2 & 4 & & & \\
\hline \multirow{2}{*}{5.} & \multirow{2}{*}{$\begin{array}{l}\text { Impulse control } \\
\text { disorder }\end{array}$} & Without illness & 11 & 69 & 18 & 98 & \multirow{2}{*}{1.373} & \multirow{2}{*}{2} & \multirow{2}{*}{0.503} \\
\hline & & With illness & 0 & 1 & 1 & 2 & & & \\
\hline \multirow{2}{*}{6.} & \multirow{2}{*}{$\begin{array}{c}\text { Borderline } \\
\text { personality traits }\end{array}$} & Without illness & 10 & 63 & 16 & 89 & \multirow{2}{*}{0.558} & \multirow{2}{*}{2} & 0757 \\
\hline & & With illness & 1 & 7 & 3 & 11 & & & 0.757 \\
\hline & Multiple psychiatric & Without illness & 11 & 67 & 18 & 96 & & & \\
\hline 7. & disorders & With illness & 0 & 3 & 1 & 4 & 0.552 & 2 & 0.7 \\
\hline & Family history of & Without family history & 8 & 66 & 18 & 92 & & & \\
\hline 8. & psychiatric illness & With family history & 3 & 4 & 1 & 8 & 6.242 & 2 & (significant) \\
\hline 9 & Family history of & Without family history & 11 & 65 & 19 & 95 & & 2 & \\
\hline 9. & suicide attempt & With family history & 0 & 5 & 0 & 5 & 2.256 & 2 & $0.3 \angle 4$ \\
\hline
\end{tabular}

\begin{tabular}{|c|c|c|c|c|c|c|c|}
\hline Sex & Dimensions of Impulsivity & Sources of Variation & Sum of Squares & df & Mean Square & $\mathbf{F}$ & Sig. \\
\hline \multirow{18}{*}{ Male } & \multirow{3}{*}{ Attention } & Between Groups & .667 & 2 & .334 & 1.729 & .189 \\
\hline & & Within Groups & 8.874 & 46 & .193 & & \\
\hline & & Total & 9.541 & 48 & & & \\
\hline & \multirow{3}{*}{ Cognitive Instability } & Between Groups & .282 & 2 & .141 & .439 & .648 \\
\hline & & Within Groups & 14.811 & 46 & .322 & & \\
\hline & & Total & 15.093 & 48 & & & \\
\hline & \multirow{3}{*}{ Motor } & Between Groups & .082 & 2 & .041 & .170 & .845 \\
\hline & & Within Groups & 11.130 & 46 & .242 & & \\
\hline & & Total & 11.212 & 48 & & & \\
\hline & \multirow{3}{*}{ Perseverance } & Between Groups & .131 & 2 & .066 & .385 & .683 \\
\hline & & Within Groups & 7.841 & 46 & .170 & & \\
\hline & & Total & 7.972 & 48 & & & \\
\hline & \multirow{3}{*}{ Self-Control } & Between Groups & 1.132 & 2 & .566 & 3.069 & .055 \\
\hline & & Within Groups & 8.480 & 46 & .184 & & \\
\hline & & Total & 9.611 & 48 & & & \\
\hline & \multirow{3}{*}{ Cognitive Complexity } & Between Groups & .135 & 2 & .068 & .262 & .771 \\
\hline & & Within Groups & 11.904 & 46 & .259 & & \\
\hline & & Total & 12.039 & 48 & & & \\
\hline & & ysis of Impulsi & ors among Ge & $(\mathrm{IV})$ & & & \\
\hline
\end{tabular}

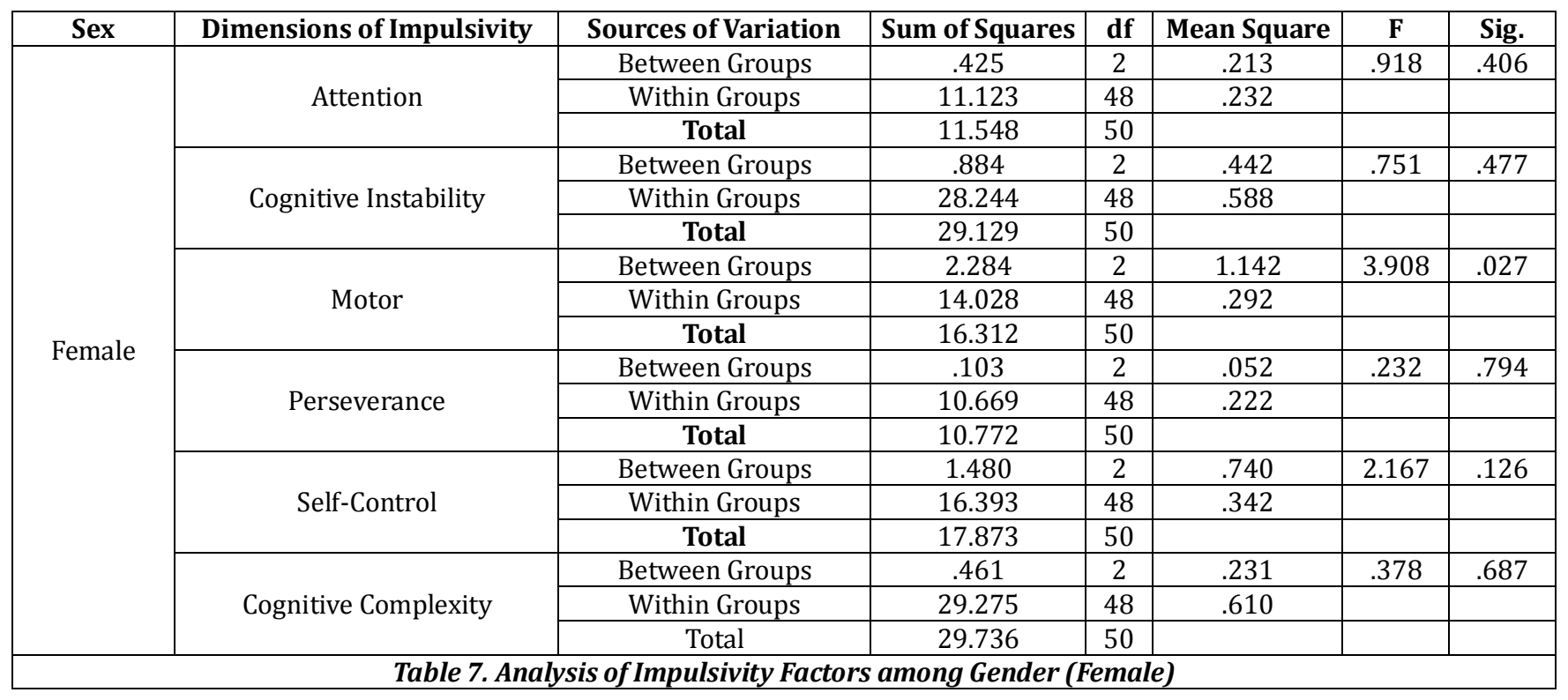




\begin{tabular}{|c|c|c|c|c|c|c|c|c|}
\hline & & Attention & \begin{tabular}{|c|} 
Cognitive \\
Instability
\end{tabular} & Motor & Perseverance & $\begin{array}{c}\text { Self- } \\
\text { Control }\end{array}$ & $\begin{array}{c}\text { Cognitive } \\
\text { Complexity }\end{array}$ & \begin{tabular}{|c|} 
Suicide \\
Intent Score
\end{tabular} \\
\hline \multirow{3}{*}{ Attention } & Pearson Correlation & 1 & $.515^{* *}$ & $.479^{* *}$ & $.531^{* *}$ & $.501^{* *}$ & $.547^{* *}$ & .015 \\
\hline & Sig. (2-tailed) & & .000 & .000 & .000 & .000 & .000 & .884 \\
\hline & $\mathrm{N}$ & 100 & 100 & 100 & 100 & 100 & 100 & 100 \\
\hline \multirow{3}{*}{$\begin{array}{l}\text { Cognitive } \\
\text { Instability }\end{array}$} & Pearson Correlation & $.515^{* *}$ & 1 & $.274^{* *}$ & $.393^{* *}$ & $.281^{* *}$ & $.317^{* *}$ & -.086 \\
\hline & Sig. (2-tailed) & .000 & & .006 & .000 & .005 & .001 & .393 \\
\hline & $\mathrm{N}$ & 100 & 100 & 100 & 100 & 100 & 100 & 100 \\
\hline \multirow{3}{*}{ Motor } & Pearson Correlation & $.479^{* *}$ & $.274^{* *}$ & 1 & $.559^{* *}$ & $.391^{* *}$ & $.346^{* *}$ & -.014 \\
\hline & Sig. (2-tailed) & .000 & .006 & & .000 & .000 & .000 & .892 \\
\hline & $\mathrm{N}$ & 100 & 100 & 100 & 100 & 100 & 100 & 100 \\
\hline \multirow{3}{*}{ Perseverance } & Pearson Correlation & $.531^{* *}$ & $.393^{* *}$ & $.559^{* *}$ & 1 & $.299^{* *}$ & $.490^{* *}$ & -.005 \\
\hline & Sig. (2-tailed) & .000 & .000 & .000 & & .002 & .000 & .961 \\
\hline & $\mathrm{N}$ & 100 & 100 & 100 & 100 & 100 & 100 & 100 \\
\hline \multirow{3}{*}{ Self-Control } & Pearson Correlation & $.501^{* *}$ & $.281^{* *}$ & $.391^{* *}$ & $.299^{* *}$ & 1 & $.404^{* *}$ & .043 \\
\hline & Sig. (2-tailed) & .000 & .005 & .000 & .002 & & .000 & .674 \\
\hline & $\mathrm{N}$ & 100 & 100 & 100 & 100 & 100 & 100 & 100 \\
\hline \multirow{3}{*}{$\begin{array}{l}\text { Cognitive } \\
\text { Complexity }\end{array}$} & Pearson Correlation & $.547^{* *}$ & $.317^{* *}$ & $.346^{* *}$ & $.490^{* *}$ & $.404^{* *}$ & 1 & .193 \\
\hline & Sig. (2-tailed) & .000 & .001 & .000 & .000 & .000 & & .054 \\
\hline & $\mathrm{N}$ & 100 & 100 & 100 & 100 & 100 & 100 & 100 \\
\hline \multicolumn{9}{|c|}{ **. Correlation is significant at 0.01 level (2-tailed) } \\
\hline
\end{tabular}

\section{DISCUSSION}

In our study, males and females are almost equal in number (51:49). About two-third of the study population were between the age of 18 - 30 yrs. (66\%). Our study population constitutes entirely of middle and lower socioeconomic group.

In analysing suicide intent among the persons with depressive disorder $(n=8), 25 \%$ expressed high suicide intent and $62.5 \%$ expressed medium suicide intent. This finding is similar to the study of K. Suominen, E. Isometsa et $\mathrm{al}^{4}$ and study done by Linehan MM. ${ }^{7}$ In persons without depressive disorder, $76 \%$ showed medium intent and $18.5 \%$ expressed high suicide intent.

In assessing suicide intent in persons with adjustment disorder with depressed mood ( $n=7), 43 \%$ expressed high suicide intent score and $57 \%$ expressed medium intent. In persons with binge drinking pattern of alcohol dependence syndrome $(n=4)$, one-fourth expressed higher intent and three-fourth expressed medium intent. While assessing persons with impulse control disorder $(n=2)$, all expressed medium intent only. In persons with borderline personality traits $(n=11)$, around three-fourth expressed medium intent and nearly one-fifth expressed high intent. In our study analysis, suicide intent between persons with individual mental illness (namely depressive disorder, adjustment disorder with depressed mood, alcohol dependence syndrome) and without mental illness showed no statistically significant difference (Table 3).

In comparing impulsivity among males and females, $63.2 \%$ males are within normal limits, $24.5 \%$ males expressed high impulsivity scores, $76.5 \%$ females are within normal limits, $13.7 \%$ females scored high on impulsivity.

On examining impulsivity among alcohol dependence syndrome individuals, 27\% expressed high impulsivity, 64\% scored within normal limits, majority of persons without alcohol dependence expressed normal to good control over impulsivity. In persons with depression $(n=8), 75 \%$ scored within normal limit, $12.5 \%$ belonged to high impulsivity group. In this study, persons with alcohol dependence expressed high impulsivity compared to individuals with depressive disorder. This finding is consistent with the study done by K Suominen and E Isometsa et al.4

In assessing correlation between factors of impulsivity and suicidal intent, factors such as attention, cognitive complexity and self-control showed positive correlation and impulsivity factors such as cognitive instability, motor and perseverance showed negative correlation. Significant correlation was found between cognitive complexity factor of impulsivity with suicide intent score (0.05) (Table 7). Study of Suominen $\mathrm{K}$ and $\mathrm{E}$ Isometsa et $\mathrm{al}^{4}$ reveals significant correlation between impulsiveness and suicidal intent.

In comparing persons without multiple psychiatric disorders $(18.75 \%)$, persons with multiple psychiatric disorders $(25 \%)$ had high suicidal intent. In patients with impulse control disorder $(n=2), 50 \%$ showed high impulsivity compared with only $18.36 \%$ of persons without impulse control.

In patients with borderline personality traits, $27 \%$ had high impulsivity compared with only $18 \%$ of persons without borderline personality traits. Analysing subjects with multiple psychiatric disorders $(n=4)$ regarding impulsiveness, $75 \%$ were in normal limits of impulsivity, $25 \%$ of them expressed high impulsivity. In persons without multiple psychiatric disorders $(n=96), 19 \%(n=18)$ expressed high impulsivity and $70 \%(n=67)$ scored within normal limits of impulsivity.

In our study, persons showing good control over their impulsivity were very less in number among persons with psychiatric illness.

\section{Implications}

Impulsiveness and intent were found to be significant factors in persons with suicide attempt. During assessment of patients with psychological problems, the above factors are to be given greater significance.

\section{Limitations}

Since the study is cross-sectional and one-time assessment of patient is done, a prospective study would able to assess the impulsivity factors in more detail. 


\section{CONCLUSION}

In conclusion, suicide attempts differ among attempters. The present findings suggest qualitative difference among gender in impulsivity factors. Suicide attempters with biological factors with respect to family history of psychiatric illness played a greater role in increasing the risk of impulsivity and suicide intent.

\section{Future Directions}

In the era of increasing suicide attempts, in spite of its social implications research work is sparse and given less importance compared to other psychiatric disorders. Along with current factors studied other factors like hopelessness, social support and interventions can be included in future studies.

\section{REFERENCES}

[1] Sudak HS. Psychiatric emergencies. In: Sadock BJ, Sadock VA, Ruiz P. eds. Kaplan \& Sadock's comprehensive textbook of Psychiatry. Vol 2. $9^{\text {th }}$ edn. Philadelphia, PA: Lippincott Williams \& Wilkins 2009:2717-2731.

[2] Gvion Y, Apter A. Suicide and suicidal behavior. Public Health Reviews 2012;34(2):1-20.

[3] Lonnqvist JK. Epidemiology and causes of suicide. In: Gelder MG, Andreasen NC, Copez-Ibor JJ, et al. eds. New Oxford Textbook of Psychiatry. Vol 1. 2nd edn. United States: Oxford University Press Inc., 2009:951-5.

[4] Suominen K, Isometsa E, Henriksson $M$, et al. Hopelessness, impulsiveness and intent among suicide attempters with depression, alcohol dependence or both. Acta Psychiatr Scand 1997;96(2):142-9.

[5] Cheng AT. Mental illness and suicide. A case-control study in East Taiwan. Arch Gen Psychiatry 1995;52(7):594-603.

[6] Beck AT, Schuyler D, Herman I. Development of suicidal intent scales. In: Beck AT, Resnick HLP, Lettieri DJ. eds. The prediction of suicide. Bowie, MD: Charles Press Publishers Inc., 1974:45-56.

[7] Linehan MM. Suicidal people: one population or two? Ann NY Acad Sci 1986;487:16-33.
[8] Pompili M, Innamorati M, Raja M, et al. Suicide risk in depression and bipolar disorder: Do impulsivenessaggressiveness and pharmacotherapy predict suicidal intent? Neuropsychiatric Disease and treatment 2008:4(I)247-55.

[9] Weishaar ME, Beck AT. Hopelessness and suicide. Int Review Psychiatry 1992;4(2):177-84.

[10] Pokorny AD, Kaplan HB, Tsai S. Hopelessness and attempted suicide: a reconsideration. Am J Psychiatry 1975:132(9):954-6.

[11] Strosahl K, Chiles JA, Linehan M. Prediction of suicide intent in hospitalized parasuicides: reasons for living, hopelessness and depression. Compr Psychiatry 1992;33(6):366-73.

[12] Evans J, Platts H, Liebenau A. Impulsiveness and deliberate self-harm: a comparison of 'first -timers' and 'repeaters'. Acta Psychiatr Scand 1996;93(5):378-80.

[13] Bechara A, Damasio H, Tranel D, et al. Deciding advantageously before knowing the advantageous strategy. Science 1997;275(5304):1293-5.

[14] Barratt ES, Stanford MS, Felthous AR, et al. The effects of phenytoin on impulsive and premeditated aggression: a controlled study. J Clin Psychopharmocol 1997;17(5):341-9.

[15] Meda SA, Stevens MC, Potenza MN, et al. Investigating the behavioural and self-report constructs of impulsivity domains using principal component analysis. Behav Pharmacol 2009;20(5-6):390-9.

[16] Smith AR, Witte TK, Teale NE, et al. Revisiting impulsivity in suicide: implications for civil liability of third parties. Behav Sci Law 2008;26(6):779-97.

[17] Joiner TE, Steer RA, Brown G, et al. Worst-point suicidal plans: a dimension of suicidality predictive of past suicide attempts and eventual death by suicide. Behaviour Research and Therapy. 2003;41(12):146980.

[18] Stanford MS, Mathias CW, Dougherty DM, et al. Fifty years of the Barratt impulsiveness scale: an update and review. Personality and Individual Differences 2009;47(5):385-95. 